\title{
3D EARTHWORK BIM DESIGN AND ITS APPLICATION IN AN ADVANCED CONSTRUCTION EQUIPMENT OPERATION
}

\author{
Jeonghwan $\mathrm{Kim}^{1}$, Heeyeon $\mathrm{Kim}^{2}$, Waqas Arshad Tanoli ${ }^{3}$ Jongwon Seo \\ ${ }^{1}$ KyungPook National University \\ 80 Daehakro, Bukgu, Daegu, Korea \\ 2,3,4 Hanyang University \\ 222 Wangsimni-ro, Seongdong-gu, Seoul, Korea \\ ${ }^{4}$ Corresponding author: jseo@hanyang.ac.kr
}

\begin{abstract}
Introduction: Advent of new technologies leads the construction industry in utilizing the modern techniques, especially in the earthwork process. The machine guidance system is one of such examples which allows the operator to work more efficiently by using the design on the display screen. Methods: This paper outlines a new Building Information Modeling (BIM) approach to earthwork design with the development of module system which calculates the actual earth volume and generates a TIN surface for machine guidance. This method has importance in generating Triangular Irregular Network (TIN) surface which can be used for visualization of the 3D model as well as for the machine guidance. Results and discussion: The integration of IT based system with the earthwork site results in higher efficiency and productivity. In earthwork process, the proper flow of information is essential as it can increase the transparency and lead to process improvement. This module provides an easy approach for the model generation with its user-friendly interface.
\end{abstract}

\section{Keywords}

BIM, Machine guidance, Earthwork, Construction equipment.

\section{Introduction}

Earthwork operations possess a significant value in any construction project and can cost about $25 \%$ for the overall project budget (Hare et al., 2011). The estimation and planning for earthwork operations directly impact the cost and duration of the project, and these operations signify the success and failure of the project, which implies that the earthwork quantity takeoff play an important role in a construction project(Smith et al., 2000). Accordingly, accurate estimation of earthwork volume is essential to assess the number of equipment, construction period, and construction cost to be used for construction. With regard to accurate estimation, various methods were used to calculate the earthwork volume at the design stage of the construction project.

In the earthwork design phase, generally, there are two conventional earthwork estimation methods; Average End Area (AEA) method and surface-to-surface method. Their estimation accuracies are, however, not compatible to the Digital Terrain Model (DTM) based estimation due to its neglecting nature of interpolation. Therefore, to obtain reliable earthwork quantity takeoff outcomes, Digital Terrain Model (DTM) based earthwork estimation system is required. Indeed, earthwork estimation software using cross section or TIN prism to calculate earthwork volume have been developed substantially. For example, Civil 3D developed by Autodesk, Key TERRA developed by KTF software, and many others are available as an automated tool. These programs have functions to conduct 3D earthwork modeling using topographic information and quantity estimation of cut and fill. Such automated softwares are now increasingly used.

In addition, in the construction phase, the advent of new technologies also leads the construction industry in utilizing the modern techniques, especially in the earthwork process. The machine guidance system is one of such examples which allows the operator to work more efficiently by using the design on the display screen. The operator can use 2D or 3D design which helps in eliminating the traditional surveying methods which can create hindrance in the smooth operations, the 3D digital terrain design model is usually used for machine guidance. These models provide the information regarding elevation and terrain design to the operator through digital screen mounted in the cabin of earthmoving equipment. Many researchers have identified the enhanced quality and productivity in the construction projects using automated machine guidance (Jonasson et al., 2002). The modeling for the machine guidance system also improves the planning for the construction process with less rework 
on the designed model (Barrett, 2008). Use of such technology provides higher accuracy with less time and reduced cost. The operator of the earthmoving equipment can effectively visualize and move around the site using equipment position on the design model (Baertlein et al., 2000).

Although 3D earthwork estimation software has been used for many projects during construction phase, to date, the seamless integration between 3D earthwork design information from the 3D earthwork estimation software and machine guidance operation has not been considered. More specifically, inputting design information into the machine guidance is unnecessarily required for the machine guidance operation. For example, the current machine guidance operation uses manual information transfer via flash drive or e-mail to utilize the design information (.dxf file) for machine operation. It causes disconnection of information between design phase and construction phase, which leads to inefficient, timeconsuming, and unnecessary operation.

This paper outlines a new Building Information Modeling (BIM) approach to earthwork design with the development of module system which calculates the actual earth volume and generates a TIN surface for machine guidance. This method has importance in generating Triangular Irregular Network (TIN) surface which can be used for visualization of the 3D model as well as for the machine guidance. Conventionally, the conversion of the $2 \mathrm{D}$ model to $3 \mathrm{D}$ is a cumbersome and tedious process (Vonderohe, 2009). The module is developed using visual basic programming and is compatible with the CAD based commercial software. Also It is capable of targeting formation level, slope generation, volume calculation, and the creation of a model for machine guidance. The machine guidance technology has replaced the staking process and labor intensive survey work (Hammad et al., 2013). This technology is primarily used for earthwork projects. However, it is also useful for other operations like paving and lifting (Kirchbach et al., 2012). The integration of IT based system with the earthwork site results in higher efficiency and productivity. In earthwork process, the proper flow of information is essential as it can increase the transparency and lead to process improvement (Peyret et al., 2000). This module provides an easy approach for the model generation with its user-friendly interface (Tanoli et al., 2017).

\section{Developing a 3D Earthwork BIM design Software}

This paper presents a new BIM-based module for earthwork operations. Visual Basic 6.0 is used as the main software language for the development of this module, and it uses Windows operating system. The system is developed in conjunction with $C A D$ engine to produce 3D shape information. This module is an independent program and compatible with the other commercial design software like Autodesk Civil 3D. This research presents the formatting level based technique for the modeling of earthwork design. It applies the volume calculation method which uses projection and interpolation for accurately calculating the earthwork volume. The earthwork design system components are presented in Figure 1.

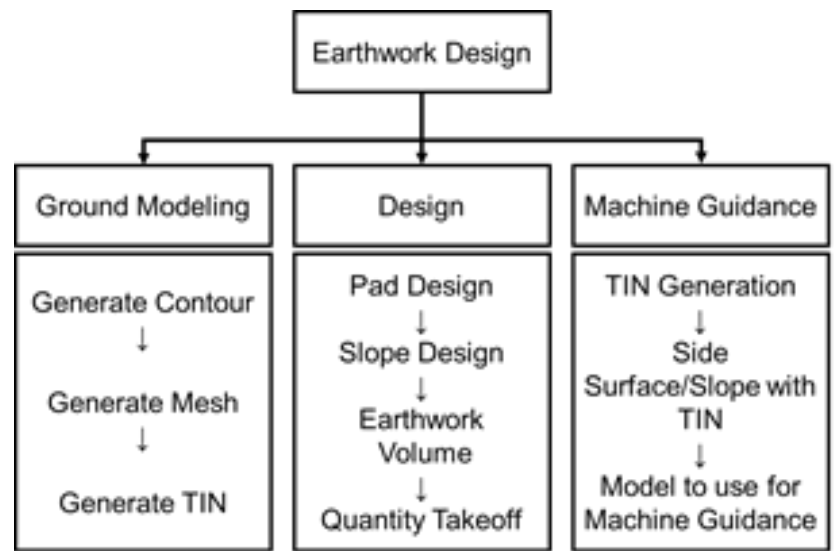

Figure 1. Earthwork design system components.

The module has simple and user-friendly graphical user interface (GUI) which is shown in Figure 2. It has import function through which ground surface in the form of contour or TIN can be directly imported. It is also capable of importing point cloud data using .mdb file and generate the ground TIN surface. The formatting level surface is created by providing the design elevation with co-ordinates information. The formatting level based design can also be drawn on the topographic model with the desired altitude value, as all the points on formatting level have the same elevation.

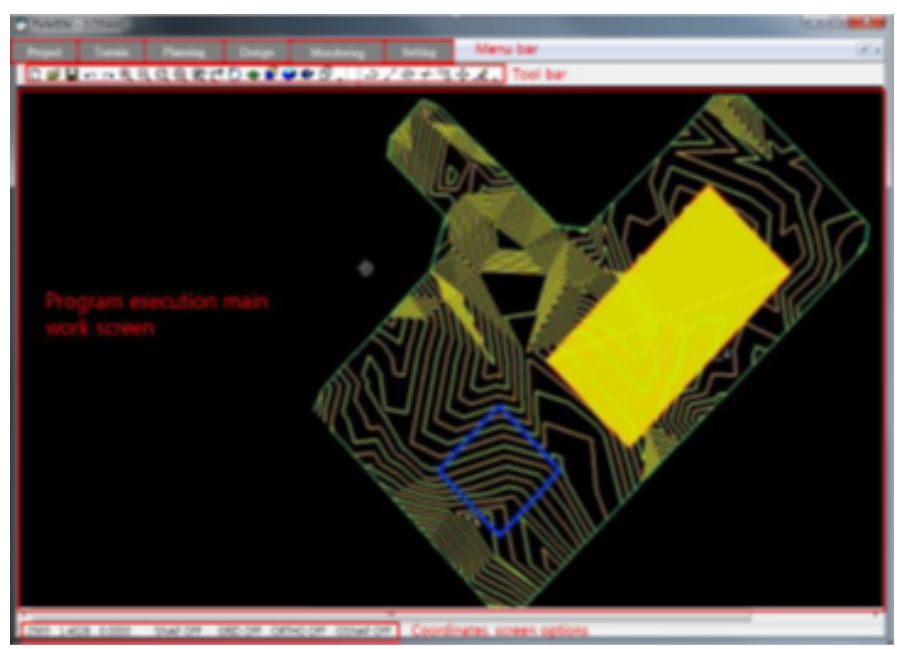

Figure 2. Module main screen.

The TIN surface is automatically created using points in the formatting level area. However, the next step in the design is to connect the formatting level with the existing ground surface for which the projection angle is required. Depending on the site requirements it is possible to generate the projection from the formatting level surface in vertical or slope form, as the system can calculate the volume in each case. The program can calculate the cut and fill volume at any given section along the length of design formatting level. The process of volume calculation is explained using flow chart in Figure 3. 


\section{Architecture and Engineering Volume 4 Issue 2}

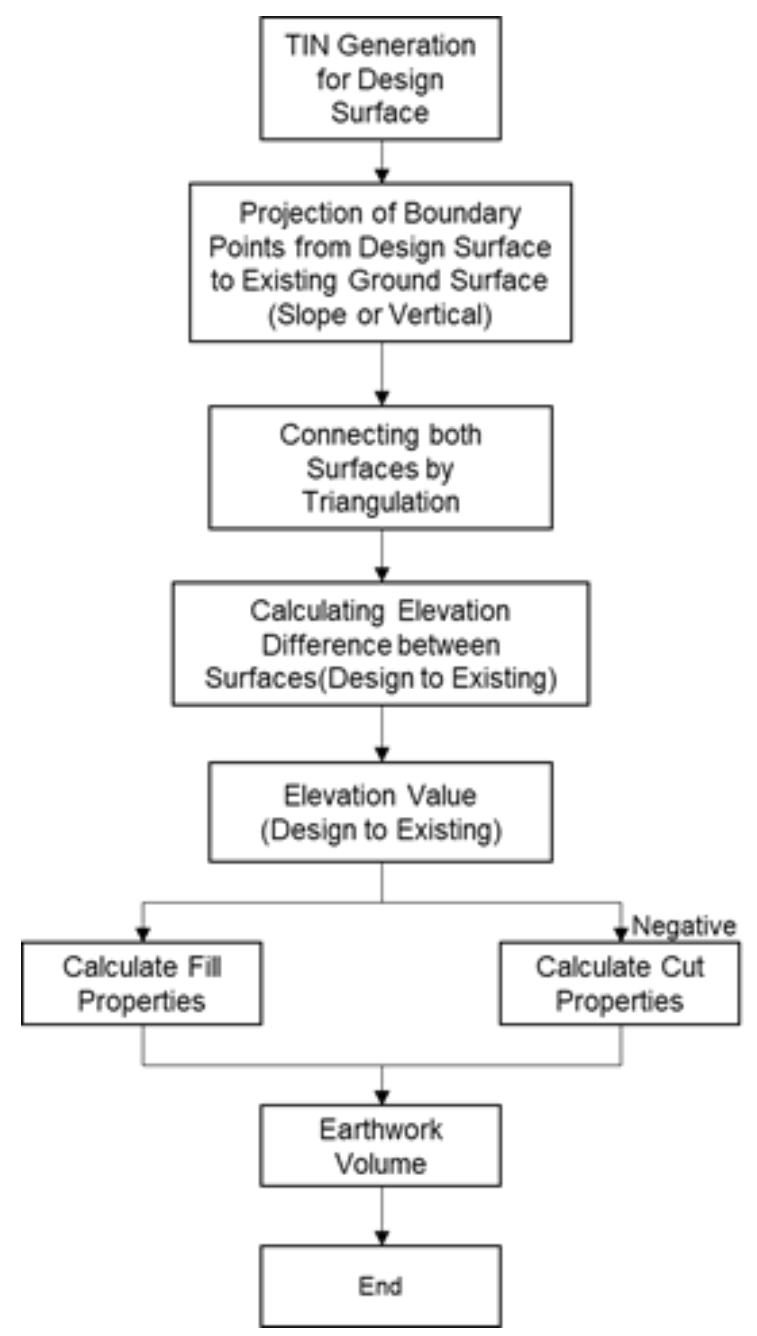

Figure 3. Flowchart for volume calculation of 3D model.

Traditionally, average end area method is used for volume calculation based on the average area between two consecutive cross sections.

$V=\frac{L}{2} \cdot\left[A_{1}+A_{2}\right]$

where $A_{1}$ and $A_{2}$ are the areas of cross-section and $L$ is the distance between these cross-sections. It is challenging to use this method continuously for the nonlinear sections (Slattery et al., 2012). However, this module used the surface to surface method for better accuracy. A new surface elevation is calculated based on the difference between two surface elevations using the location where the edges of triangles intersect the two surfaces as shown in Figure 4.

It is important to know the elevation difference between existing and planned ground surfaces. The existing and proposed ground TIN surfaces are created using survey and design data. In the case of the formatting level projection with no slope to the existing ground surface, the triangular network for side wall is generated between two surfaces. In this case, ground and design model share the same $(X Y)$ coordinates with the only difference of elevation.

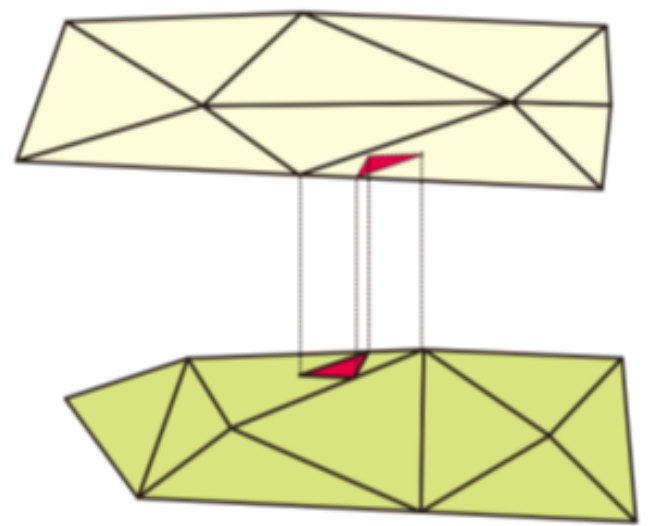

Figure 4. Surface projection illustration.

By using $\mathbf{Z}$ (elevation) value, the quantity can be estimated by triangular columns volume. If the formatting level elevation value " $Z$ " is lower than the ground TIN surface the volume is calculated as Cut, however, if the designed formatting level is located at a higher elevation than the ground surface, the fill volume is calculated.

A polyline is drawn along the formatting level surface in a longitudinal direction from one end to the other for dividing the area into equidistance stations. The module also provides the equipment monitoring function. The design drawings in the module are saved in a .dwg file format which is converted into a .dxf file using Autodesk Civil 3D. The 3D design model with DTM (Digital Terrain Model) is then available for use in the machine guidance system.

\section{Case Study: Integration to the Machine guidance}

The following case study aims to check the practical application and the validity of developed module system. The model is designed using the formatting level based module and is verified on site for use in machine guidance system.

A site in Seoul, South Korea is selected as a pilot project to test the developed module system. The area under study is not in regular rectangular shape. The site topographic data is collected using laser scanning, the 3D laser scanning technology provides higher density data as compared to traditional surveying methods. Ground contour data is imported into the module and using its TIN generation function the surface is converted into TIN. Figure 5 presents the original ground surface model in contour and TIN form.

A rectangular area is selected, and formatting level design is drawn on the BIM-based topographic model. The formatting level dimensions are based on required length and width at the site. The design altitude value is then provided to the formatting level, as the whole surface have same elevation value.

According to design and site requirements, it is possible to design in slope and vertical form, and the system is capable of calculating the volume in both cases. Figure 6 shows a formatting level design with the slope, projecting to the existing ground surface. The outer boundary is 


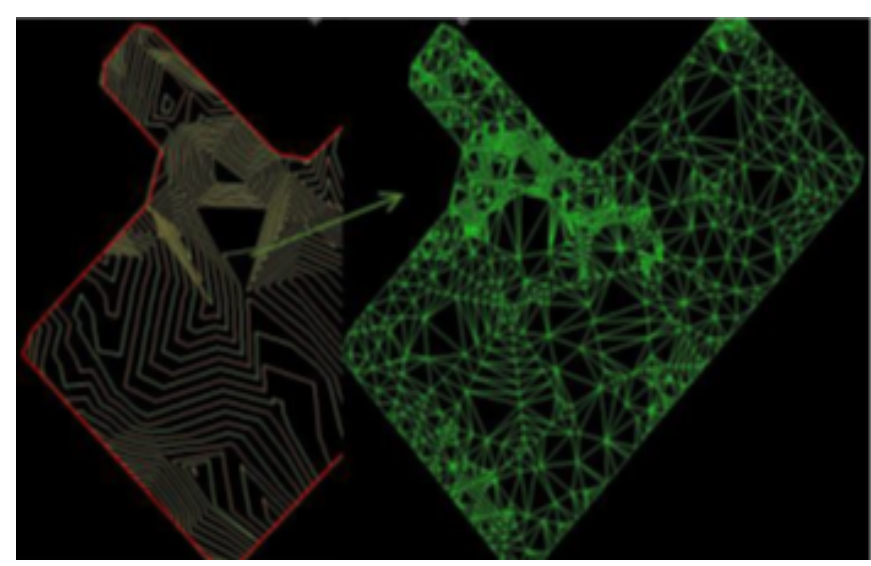

Figure 5. Ground surface model in contour and TIN form.

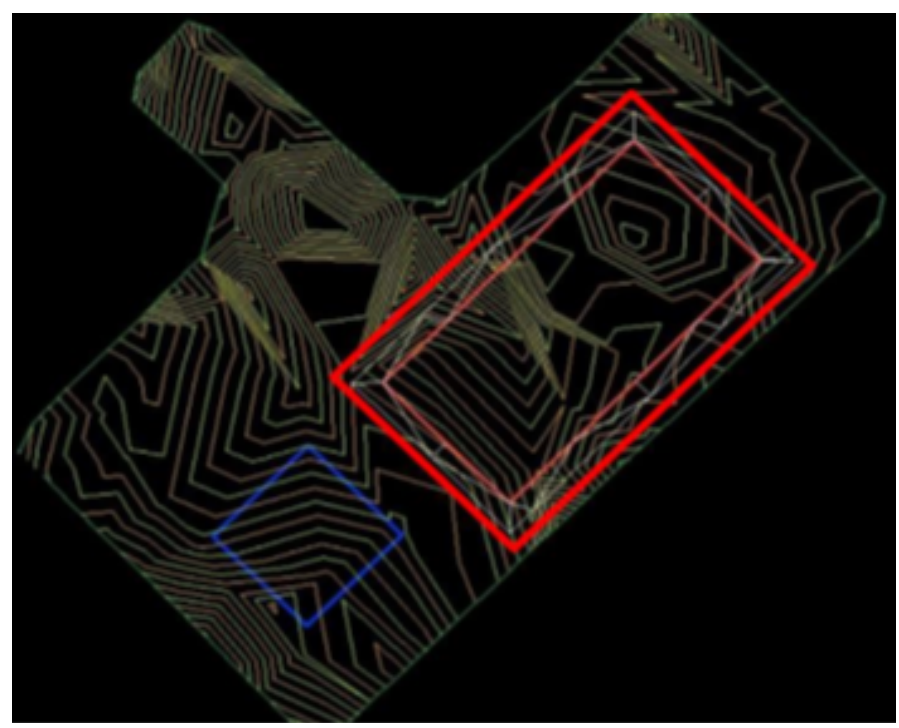

Figure 6. Formatting level based design with existing ground surface.

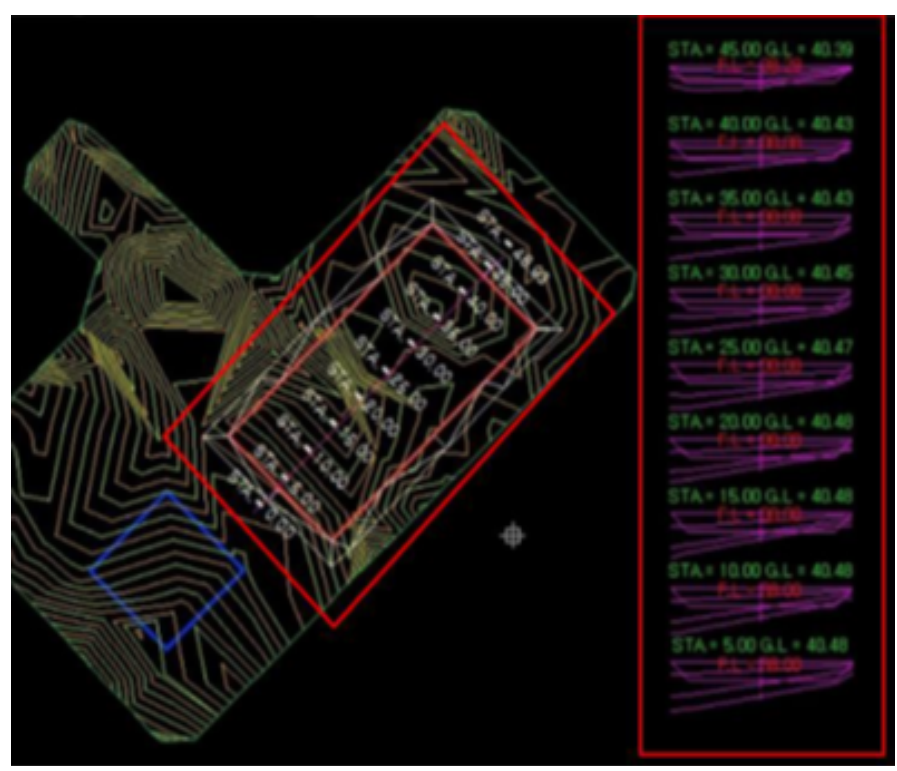

Figure 7. Formatting level design with station information.

indicating the connection with the existing ground surface, and inner rectangular marking depicts the formatting level design surface.

The formatting level design surface is then divided into stations along its length as shown in Figure 7. The ground

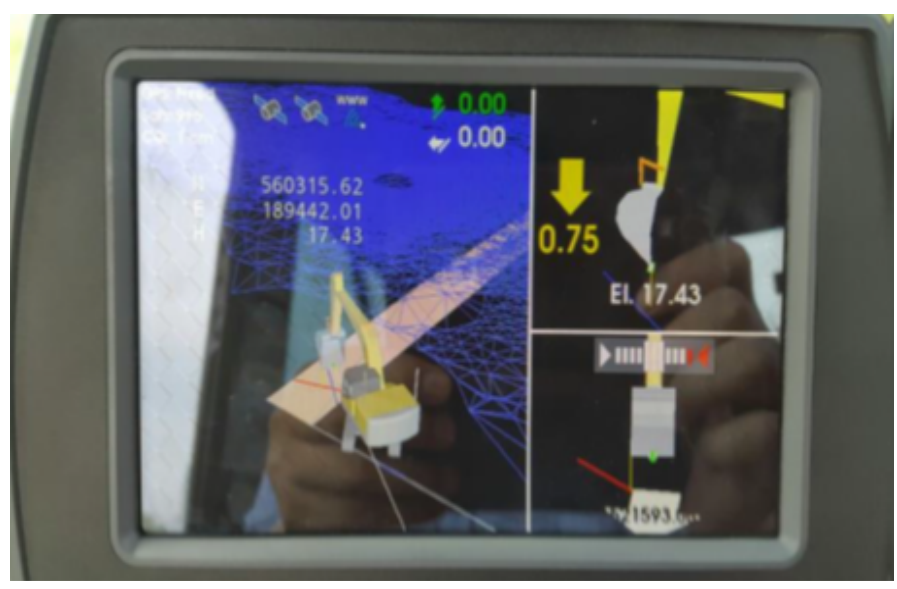

Figure 8. View of machine control panel.

level elevation can be seen at any station. It provides the function to divide the formatting level into stations with equal or different length. The formatting level based design file having TIN surface is imported in Autodesk Civil 3D and saved in .dxf file format. The machine guidance control panel requires the TIN file in .dxf file format.

The files with TIN surface are only visible in the control panel of earthmoving equipment. The formatting level design file is then imported into the machine control panel. Figure 8 shows the view of machine control panel for the operator in the earthmoving equipment.

\section{Conclusion and Future Study}

This paper outlines a new Building Information Modeling (BIM) approach to earthwork design with the development of module system which calculates the actual earth volume and generates a TIN surface for machine guidance. Visual Basic 6 is used for development of this module in conjunction with the CAD engine. The topographic modeling function supports 3D BIM design using the point cloud data obtained from laser scanning. It is possible to draw and design a formatting level on the 3D ground with consideration of slope and vertical planning. For the volume calculation of cut or fill in between formatting level design surface and existing ground, this module used the surface to surface method for better accuracy.

The developed module is capable of creating a TIN model for use in machine guidance system. A case study has proved the validity and effectiveness of this module for earthwork design. The module at this time can only deal with TIN models, however, with the integration of algorithm for solid and parametric modeling technique, its usability will increase.

Further studies are in progress to update this formatting level based BIM module, incorporating the 4D (time) and 5D (cost) design functions to automate the calculation of time and cost at any particular section. The large scale validity assessment will also be carried out in projects with different cost and duration.

\section{Acknowledgement}

This work was supported by the National Research Foundation of Korea (NRF) grant funded by the Korea government (MSIT) (No. NRF-2018R1A5A1025137). 


\section{Architecture and Engineering Volume 4 Issue 2}

\section{References}

Baertlein, H., Carlson, B., Eckels, R., Lyle, S., Wilson, S. (2000). A High-Performance, High-Accuracy RTK GPS Machine Guiadance System. GPS Solutions, 3 (3), pp. 4-11. DOI: 10.1007/PL00012802.

Barrett, L. (2008). Automated Machine Guidance: Emerging Technology Whose Time Has Come? In: Proceedings 87th Annual Meeting of Transportation Research Board. Washington DC, United States.

Hammad, A., Vahdatikhaki, F., Zhang, C. (2013). A novel integrated approach to project-level automated machine control/guidance systems in construction projects. Journal of Information Technology in Construction, 18 (9), pp. 162-181.

Hare, W. L., Koch, V. R., Lucet, Y. (2011). Models and algorithms to improve earthwork operations in road design using mixed integer linear programming. European Journal of Operational Research, 215 (2), pp. 470-480. DOI: 10.1016/j.ejor.2011.06.011.

Jonasson, S., Dunston, P. S., Ahmed, K., Hamilton, J. (2002). Factors in Productivity and Unit Cost for Advanced Machine Guidance. Journal of Construction Engineering and Management, 128 (5), pp. 367-374. DOI: 10.1061/(ASCE)0733-9364(2002)128:5(367).

Kirchbach, K., Bregenhorn, T., Gehbauer, F. Digital allocation of production factors in earth work construction. In: Proceedings of the 20th Conference of the International Group for Lean Construction.

Peyret, F., Jurasz, J., Carrel, A., Zekri, E., Gorham, B. (2000). The computer integrated road construction project. Automation in Construction, 9 (5-6), pp. 447-461. DOI: 10.1016/S0926-5805(00)00057-1.

Slattery, K. T., Slattery, D. K., Peterson, J. P. (2012). Road Construction Earthwork Volume Calculation Using Three-Dimensional Laser Scanning. Journal of Surveying Engineering, 138 (2), pp. 96-99. DOI: 10.1061/(ASCE)SU.1943-5428.0000073.

Smith, S. D., Wood, G. S., Gould, M. (2000). A new earthworks estimating methodology. Construction Management \& Economics, 18 (2), pp. 219-228. DOI: 10.1080/014461900370843.

Tanoli, W. A., Raza, H., Lee, S. S., Seo, J. W. PAD Based 3D Earthwork BIM Design Module for Machine Guidance. In: Proceedings of the International Symposium on Automation and Robotics in Construction, Vilnius Gediminas Technical University, pp. 626-631.

Vonderohe, A. (2009). Implementation of GPS controlled highway construction equipment-phase III. Madison: National Center for Freight and Infrastructure Research and Education. 